\title{
Development techniques for nano-biosensors
}

\begin{abstract}
We describe development techniques for nanowire-based nano-biosensors. We synthesized zinc oxide nanowires in a custom ambient Chemical Vapor Deposition (CVD) system. We performed characterizations on synthesized nanowires and functionalized them to experiment with immobilization of carboxylic acid moieties on the surface of nanowires for biosensing applications. We analyzed modified and unmodified zinc oxide $(\mathrm{ZnO})$ nanowires with oleic acid to sense biomolecules along with the sensing mechanism.
\end{abstract}

\author{
Volume 7 Issue | - 202| \\ Bruce C Kim,' Anurag Gupta,' Jong W Park \\ 'City University of New York, USA \\ ${ }^{2}$ Korean Research Institute of Ships \& Ocean Engineering \\ (KRISO), Korea
}

Correspondence: Bruce C Kim, City University of New York USA, Tel 20I-577-4605, Email bkim@ccny.cuny.edu

Received: January 06, 2021 | Published: January 19, 2021

\section{Introduction}

Conventionally synthesized zinc oxide nanowires are biocompatible for in vivo applications. It has been established that one-dimensional semiconductor surfaces have active sites that can be easily immobilized with many different biomolecular agents. ${ }^{1,2}$ The changes of resistive detection in chemical and biological agents are based on electrical conductivity changes on a functionalized nanowire surface. There have been several reports on the use of $\mathrm{ZnO}$ nanowires for the biological sensing of complex molecules. ${ }^{3-6}$ Our previous work has confirmed that chemical interaction is possible between oleic acid and $\mathrm{ZnO}$ nanowires. ${ }^{7}$ In this work, we demonstrate the immobilization of oleic acid molecules on a $\mathrm{ZnO}$ nanowire surface and then we experiment with a local chemical bonding environment of oleic acid molecules on a nanowire surface. Oleic acid has been shown to attract different moieties of surfactant present in biologically important molecules. ${ }^{8}$ Figure 1 shows a schematic for the idea of oleic acid binding to zinc oxide nanowire for surface modifications. Hence, in this work we synthesized $\mathrm{ZnO}$ nanowires in an ambient $\mathrm{CVD}$ furnace and established the morphology and composition of the synthesized nanowires.

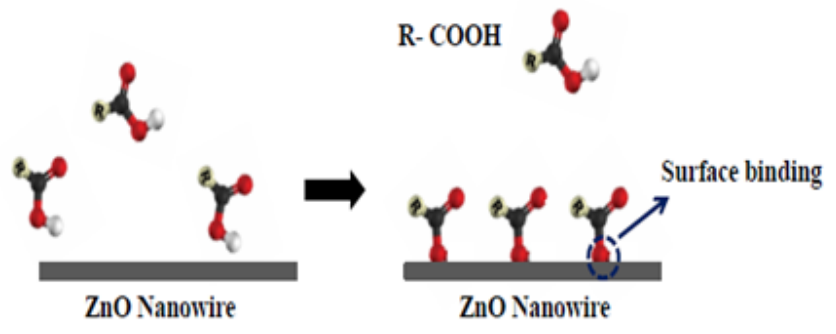

Figure I Nanowire-based detection scheme.

\section{Approach}

Zinc oxide substrate (001) has been utilized in the literature to synthesize nanowires with an ambient CVD system $.9,10$ Here, $\mathrm{ZnO}$ substrates were spin-coated with gold through RF sputtering. Zinc oxide powders and graphite were homogenously mixed in a 1:1 ratio and put into the CVD quartz tube. A mixed gas of argon and oxygen was utilized as a carrier medium at the optimum flow rate for 30 minutes. The synthesized nanowires were characterized using SEM and TEM for physical structure and morphology. The nanowires on the $\mathrm{ZnO}$ substrate were then functionalized in an oleic solution $(1 \% \mathrm{v} / \mathrm{v}$ in Hexane) for approximately 10 minutes (maximum of $15 \mathrm{~min}$.) to functionalize the nanowire surface. The functionalized nanowires were rinsed in pure hexane to remove any oleic acid that was not adsorbed to the surface of nanowires. It is critical to remove any oleic acid that has not adsorbed to prevent spurious signal generation. The photoluminescence spectrum of the unmodified and modified zinc oxide nanowires was obtained using a spectrophotometer in a quartz cuvette.

\section{Results}

Figures $2 \mathrm{~A} \& 2 \mathrm{~B}$ show the SEM images of $\mathrm{ZnO}$ nanowires on $\mathrm{ZnO}$ substrate as synthesized by the CVD system. It can be observed that the nanowires are vertically aligned with respect to the substrate and are laterally branched. Figure $2 \mathrm{~A}$ shows the vertical nanowire growth due to the absence of lattice mismatch between the nanowires and the substrate; the hierarchical growth in Figure $2 \mathrm{~B}$ could be due to the high super saturation of zinc vapor in the quartz tube, as suggested by Zhang et al. ${ }^{11}$ We performed XRD spectrum characterizations of $\mathrm{ZnO}$ nanowires, which indicated a higher peak in intensity at the preferential growth along the [0001] direction than other peaks. We observed [0001] images through TEM analysis of the nanowires, as presented in Figure 3. The figure shows the high-resolution TEM image of a selected area on the $\mathrm{ZnO}$ nanowire, which conforms to the empirical observations of $\mathrm{ZnO}$ nanowires' tendency towards a $c$-axis $(<0001>)$ growth direction via a VLS process and typical inter planar spacing of $0.52 \mathrm{~nm}$. The inset in Figure 3 shows a schematic of a wurtzite-type crystal structure of $\mathrm{ZnO}$ nanowires that typically results from a VLS growth process.

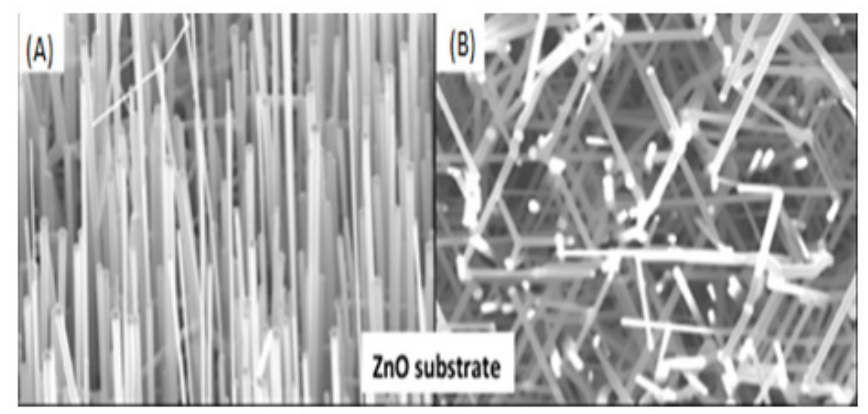

Figure $2 \mathrm{ZnO}$ nanowires on $\mathrm{ZnO}(000 \mathrm{I})$ substrate in inclined $(\mathrm{A})$ and top view $(B)$, respectively.

\section{Characterization of oleic acid nanowires}

To ascertain the chemical functionalization of oleic acid on a $\mathrm{ZnO}$ nanowire surface, Raman spectroscopy was conducted on pristine 
and oleic acid-functionalized $\mathrm{ZnO}$ nanowires. Figure 4 illustrates the Raman spectrum of the pristine $\mathrm{ZnO}$ nanowires as synthesized on $\mathrm{Si}$ (100) substrates. This was crucial to obtain and establish the necessary background. As shown in the figure, peaks at $437 \mathrm{~cm}^{-1}$ and $577 \mathrm{~cm}^{-1}$ are characteristic to $\mathrm{ZnO}$. While the relative intensity of the peak at $437 \mathrm{~cm}^{-1}$ for single crystalline $\mathrm{ZnO}$ should be higher than for any other characteristic $\mathrm{ZnO}$ peaks, it can be observed from the red curve in Figure 4 that the opposite is true for the sample. This is because of the absence of a well-defined scattering configuration due to randomly oriented nanowires on the $\mathrm{Si}(100)$ substrates. This provides a slight polycrystalline nature to the sample, where a relatively prominent $437 \mathrm{~cm}^{-1}$ peak is indicative of a mixture of orientations present within the laser spot during the experiment. This variation in characteristic peaks can be seen in the images of sampling areas adjacent to the spectra. The remaining peaks, including the prominent peak at $520 \mathrm{~cm}$ ${ }^{1}$, correspond to the $\mathrm{Si}(100)$ substrate. It was also observed that the low-intensity substrate peaks at $301 \mathrm{~cm}^{-1}$ and $966 \mathrm{~cm}^{-1}$ are obscured as the sampling area for obtaining the spectra changes from a region of sparse $\mathrm{ZnO}$ nanowire growth (curve 1) to a dense $\mathrm{ZnO}$ nanowire growth (curve 6).

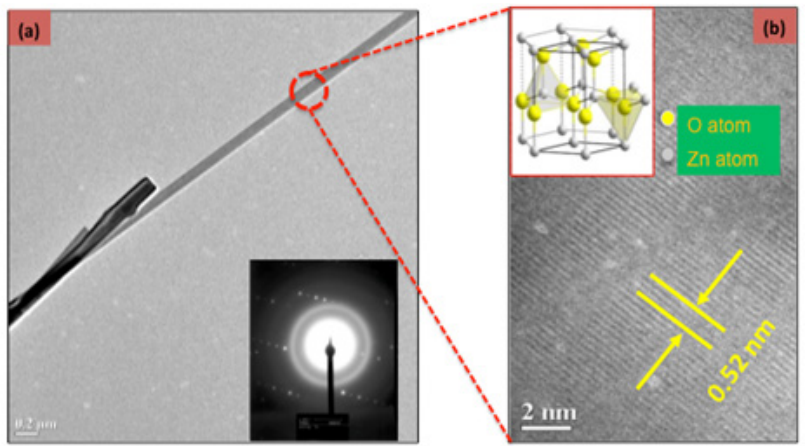

Figure 3 (A) Low-magnification TEM of a single $\mathrm{ZnO}$ nanowire; (inset) selected area electron diffraction (SAED) pattern of $\mathrm{ZnO}$ nanowire elucidating its single crystal nature, (B) HR-TEM image of a selected area on $\mathrm{ZnO}$ nanowire. The lattice spacing of $0.52 \mathrm{~nm}$ along the [000I] direction confirms the crystal structure of $\mathrm{ZnO}$ nanowire as wurtzite; (inset) schematic of wurtzite-type $\mathrm{ZnO}$.

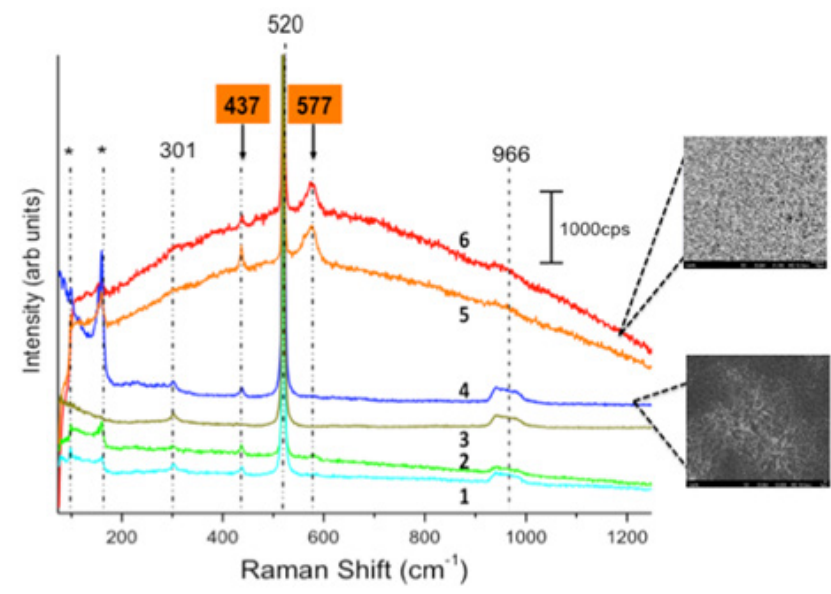

Figure 4 Raman spectrum of pristine $\mathrm{ZnO}$ nanowires on $\mathrm{Si}$ (100) substrates, taken at various sampling areas. Curve I represents Raman spectra taken in a region of thin nanowire growth, while curve 6 represents a region of dense nanowire growth.

Figure 5 illustrates the Raman spectrum of functionalized $\mathrm{ZnO}$ nanowires. It was observed that in addition to the characteristic peaks of $\mathrm{ZnO}$, signature oleic acid peaks were also present. This strongly suggests that the $\mathrm{ZnO}$ nanowire surfaces were modified by oleic acid. However, to establish chemical functionalization, Raman measurements alone provide necessary but insufficient empirical evidence. Accordingly, FT-IR spectra of oleic acid-modified nanowire samples were obtained. In Figure 5A, the characteristic $\mathrm{ZnO}$ peaks were observed at $439 \mathrm{~cm}^{-1}\left(\mathrm{E}_{2}\right.$ mode $)$ and $580 \mathrm{~cm}^{-1}\left(\mathrm{~A}_{1(\mathrm{LO})}\right.$ mode $)$, which serve as a reference for further modification procedures. ${ }^{12}$ The figure indicates that the spectrum has peaks corresponding to oleic acid in addition to characteristic zinc oxide peaks. This behavior is indicative of a possible modification of the nanowire surface by oleic acid; however, the nature of the carboxylate bonding may be ambiguous.

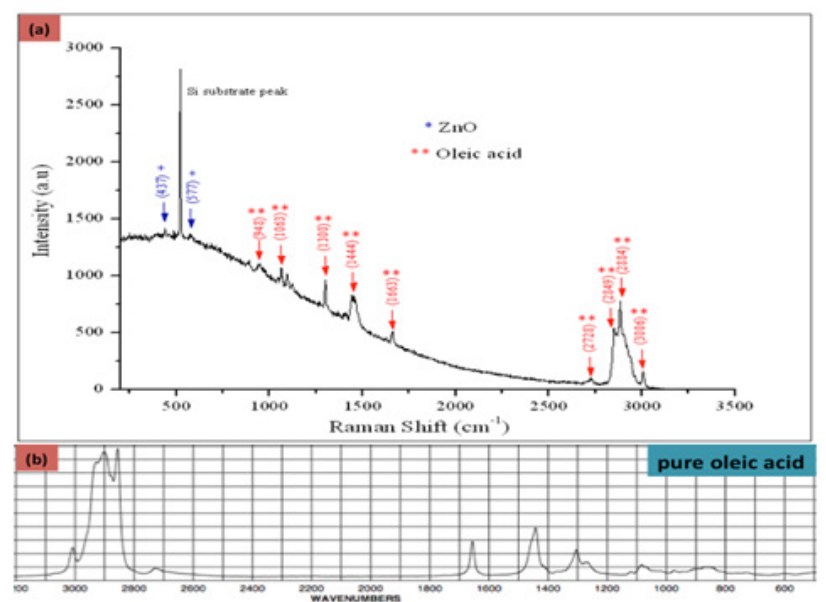

Figure 5 (A) Raman spectrum of oleic acid-modified $\mathrm{ZnO}$ nanowires, and (B) Raman spectra of pure oleic acid, indicating prominent vibration modes.

Furthermore, Figure 6 is the FT-IR spectrum of oleic acid in hexane. For oleic acid-modified nanowire, small peaks at $937 \mathrm{~cm}^{-1}$ and $1464 \mathrm{~cm}^{-1}$ correspond to out-of-plane and in-plane $\mathrm{OH}$ deformations, respectively. The major peak at $1710 \mathrm{~cm}^{-1}$ is $\mathrm{C}=\mathrm{O}$. Smaller peaks at $2570 \mathrm{~cm}^{-1}$ and $2673 \mathrm{~cm}^{-1}$ indicate oleic acid dimer formation. The group of peaks from $2825 \mathrm{~cm}^{-1}$ to $2950 \mathrm{~cm}^{-1}$ are from $\mathrm{CH}_{2}$ and $\mathrm{CH}_{3}$ asymmetric and symmetric vibration stretches. From the figure, we observe that the changes in the spectrum are primarily due to features associated with the carboxylic acid group. The remaining unidentified peaks are characteristic to long hydrocarbon chains. All peaks beyond $2800 \mathrm{~cm}^{-1}$ are associated with $\mathrm{CH}_{3}$ and $\mathrm{CH}_{2}$ as well as $\mathrm{C}=\mathrm{C}$ modes. After the functionalization of oleic acid molecules on the $\mathrm{ZnO}$ nanowire surfaces, the photoluminescence (PL) behavior of the unmodified and modified $\mathrm{ZnO}$ nanowires was tested. Figure 7A shows the PL spectrum of the pristine nanowires. Weak peaks at $510 \mathrm{~nm}$ and $536 \mathrm{~nm}$ were attributed to defect-related emission, and prominent peaks at $360 \mathrm{~nm}$ and $380 \mathrm{~nm}$ are due to the onset of excitonic band edge-related emission. When the nanowire surface sites are occupied by oleic acid molecules, two significant changes occur in the spectrum. In Figure 7B the defect-related peaks disappear, which could be due to the occupation of surface sites by oxygen from oleic acid that otherwise would have contributed to the visible luminescence. Furthermore, the band-edge related emission peaks merge and exhibit a slight shift, which can be attributed to the surface adsorption of oleic acid molecules. Although the role of oxygen in $\mathrm{ZnO}$ PL is still debated, it can nonetheless serve as a functional tool for quantification and characterization. It also highlights the importance of controlling the surface chemistry of synthesized $\mathrm{ZnO}$ nanowires for nanosensor fabrication based on organic-inorganic heterostructures. 


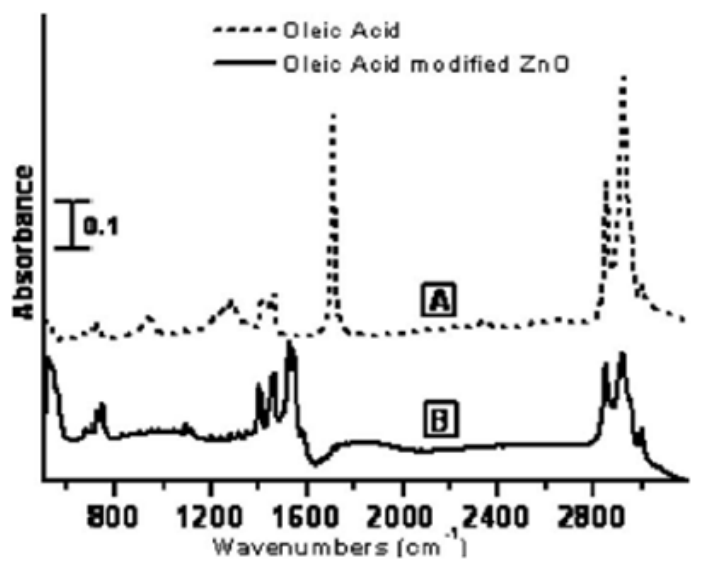

Figure 6 FT-IR spectra of (A) oleic acid I\% (v/v) in hexane; (B) oleic acidmodified $\mathrm{ZnO}$ nanowires.

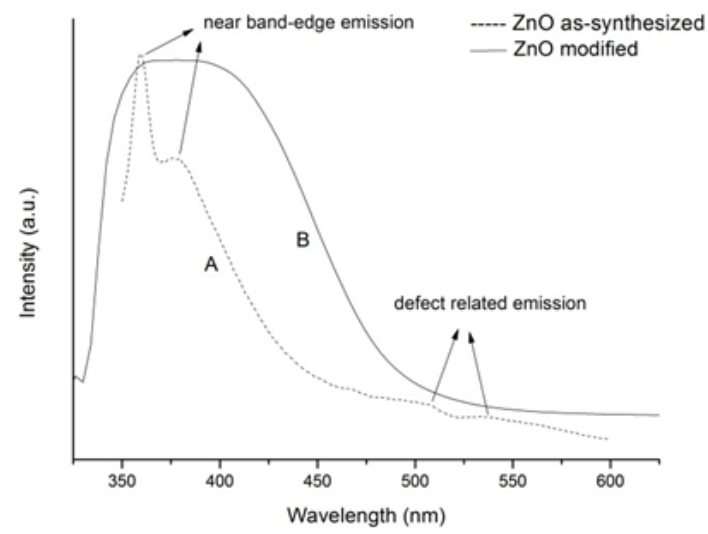

Figure 7 Photoluminescence spectrum of (A) pristine $\mathrm{ZnO}$ nanowires, and (B) oleic acid-functionalized $\mathrm{ZnO}$ nanowires [100]. The excitation wavelength was $325 \mathrm{~nm}$, with an accuracy of $0.5 \mathrm{~nm}$ and repeatability of $0.1 \mathrm{~nm}$.

\section{Conclusion}

We have described development techniques for nano-biosensors. We have synthesized nanowires in a chemical vapor deposition system, and we then functionalized them with oleic acid. We have functionalized nanowires with oleic acid to study the possibility of a biosensing application using zinc oxide nanowires. We performed Raman and infrared spectroscopy and observed that oleic acid binds to the $\mathrm{ZnO}$ nanowire surface through carboxylic acid moiety. The photoluminescence spectrum of zinc oxide nanowires showed changes in defect-related emission intensity. These characterization techniques could be a good methodology to identify differences in biological specimens. Therefore, careful surface chemistry is a key factor in developing nano-biosensors.

\section{Acknowledgements}

This work is partially supported by the project "Development of automatic screening and hybrid detection system for hazardous material detecting in port containers" from the Korean Ministry of Oceans and Fisheries (KMOF).

\section{Conflicts of interest}

The authors declare that there is no conflict of interest.

\section{References}

1. Lee S, Choi A, Kim CJ, et al. P-type Si-nanowire-based Fieldeffect Transistors for Electric Detection of a Biomarker: Matrix Metalloproteinase-9. JKPS. 2009;55:232

2. Hernandez-Velez M. Nanowires and 1D arrays fabrication: An overview. Thin Solid Films. 2006;495:51-63.

3. Hou X, Zhou F, Yu B, et al. Superhydrophobic zinc oxide surface by differential etching and hydrophobic modification. Mater Sci. 2007;732:452-453.

4. Singh SP, Sunil K Arya, Pratibha Pandey, et al. Cholesterol biosensor based on rf sputtered zinc oxide nanoporous thin film. Appl Phys Letts. 2007;91:063901

5. Yakimova R, Petoral Jr RM, Yazdi GR, et al. Surface functionalization and biomedical applications based on SiC. Biosens Bioelectron. 2007;22:2780

6. Jeon KA. Biosensors with oxide nanowires. 5th IEEE Sensors Conference. IEEE; 2006

7. Gupta A, Kim B, Watkins C, et al. Surface Chemistry for ZnO Nanowires for Nanosensor Applications. Journal of Nanotechnology in Engineering and Medicine. 2011;2:011010-1-4.

8. Mahmood I, Guo C, Xia H, et al. Lipase Immobilization on Oleic AcidPluronic (L-64) Block Copolymer Coated Magnetic Nanoparticles, for Hydrolysis at the Oil/Water Interface. Ind Eng Chem Res. 2008;47:63796385 .

9. Chang PC, Fan Z, Lu JG, et al. ZnO Nanowires Synthesized by Vapor Trapping CVD Method. Chem Mat. 2004;16:5133-5137.

10. Lee CY, Tseng TY, Li SY, et al. Growth of Zinc Oxide Nanowires on Si (100). Tamkang Journal of Science and Engineering. 2003;6:127-132.

11. Zhang Z, Wang SJ, Yu T, et al. Controlling the growth mechanism of ZnO nanowires by selecting catalyst. J Phys Chem C. 2007;111:1750017505 .

12. Huang Y, Meidong Liu, ZhenLi, et al. Raman spectroscopy study of $\mathrm{ZnO}-$ based ceramic films fabricated by novel sol-gel process. Materials Science and Engineering B. 2003;97:111-116. 